\title{
Age at menarche and risk of type 2 diabetes among African- American and white women in the Atherosclerosis Risk in Communities (ARIC) study
}

\author{
J. G. Dreyfus • P. L. Lutsey • R. Huxley • J. S. Pankow • \\ E. Selvin • L. Fernández-Rhodes • N. Franceschini • \\ E. W. Demerath
}

Received: 26 March 2012 / Accepted: 23 May 2012 / Published online: 4 July 2012

(C) Springer-Verlag 2012

\begin{abstract}
Aims/hypothesis We examined race differences in the association between age at menarche and type 2 diabetes before and after adjustment for adiposity.

Methods We analysed baseline and 9-year follow-up data from 8,491 women ( $n=2,505$ African-American, mean age 53.3 years; $n=5,986$ white, mean age 54.0 years) in the Atherosclerosis Risk in Communities (ARIC) study. Stratifying by race, we used logistic regression to estimate the OR for prevalent diabetes at baseline, and Cox proportional hazard models to estimate the HR for incident diabetes over follow-up according to age at menarche category $(8-11,12$, 13,14 and $15-18$ years).

Results Adjusting for age and centre, we found that early age at menarche (8-11 vs 13 years) was associated with diabetes for white, but not African-American women in both the prevalent (white OR 1.72, 95\% CI 1.32, 2.25; African-
\end{abstract}

Electronic supplementary material The online version of this article (doi:10.1007/s00125-012-2616-z) contains peer-reviewed but unedited supplementary material, which is available to authorised users.

J. G. Dreyfus $(\bowtie) \cdot$ P. L. Lutsey • R. Huxley • J. S. Pankow

E. W. Demerath

Division of Epidemiology and Community Health,

School of Public Health, University of Minnesota,

1300 South Second St, Suite 300,

Minneapolis, MN 55454-1015, USA

e-mail: gora0001@umn.edu

E. Selvin

Department of Epidemiology, Johns Hopkins Bloomberg

School of Public Health,

Baltimore, MD, USA

L. Fernández-Rhodes $\cdot$ N. Franceschini

Department of Epidemiology, UNC Gillings Global

School of Public Health,

Chapel Hill, NC, USA
American OR 1.13, 95\% CI 0.84, 1.51; interaction $p=$ 0.043 ) and incident models (white HR 1.43, 95\% CI 1.08, 1.89; African-American HR 1.20, 95\% CI 0.87, 1.67; interaction $p=0.527$ ). Adjustment for adiposity and lifestyle confounders attenuated associations for prevalent (white OR 1.41, 95\% CI 1.05, 1.89; African-American OR 0.94, 95\% CI 0.68, 1.30; interaction $p=0.093$ ) and incident diabetes (white HR 1.22, 95\% CI 0.92, 1.63; African-American HR 1.11, 95\% CI 0.80, 1.56; interaction $p=0.554$ ).

Conclusions/interpretation Early menarche was associated with type 2 diabetes in white women, and adulthood adiposity attenuated the relationship. We did not find a similar association in African-American women. Our findings suggest that there may be race/ethnic differences in the influence of developmental factors in the aetiology of type 2 diabetes, which merit further investigation.

Keywords Diabetes mellitus · Epidemiology $\cdot$ Growth and development $\cdot$ Menarche $\cdot$ Obesity $\cdot$ Puberty $\cdot$ Risk factor

\author{
Abbreviations \\ ARIC Atherosclerosis Risk in Communities \\ NHS Nurses' Health Study \\ SES Socioeconomic status
}

\section{Introduction}

Mounting evidence suggests that early age at menarche raises the risk for type 2 diabetes although the extent to which the association is independent of adiposity and is present in non-white populations remains unclear [1-4]. The association between early menarche and diabetes has implications for public health because of evidence that the 
average age at menarche has declined in recent years $[5,6]$, while at the same time the prevalence of obesity and diabetes have increased [7]. While it is not clear whether the increasing prevalence of early childhood obesity is causally responsible for the downward trend in age at menarche [8, 9], it is well established that early menarche is associated with higher BMI in adolescence and early adulthood [1, 2, 10-13], as well as greater waist circumference [14]. Earlier menarche may also initiate adipose tissue accumulation during later stages of development $[15,16]$, and higher abdominal obesity likely increases circulating levels of insulin, testosterone and insulin-like growth factor 1 [17]. These same mechanisms might be responsible for the elevated risk for type 2 diabetes later in life. Therefore, proper interpretation of an association between menarche timing and type 2 diabetes requires rigorous consideration of its independence from the relationship between menarche and adiposity.

Race differences in the association of age at menarche and diabetes have not been explored. Considering the health disparities associated with race/ethnicity, this leaves an important gap in the existing literature. Currently, AfricanAmerican women have, on average, a higher risk of type 2 diabetes, higher adiposity and earlier age at menarche than white women in the USA [18-20], but lower visceral fat mass relative to total adiposity than white women [21].

Using data from the Atherosclerosis Risk in Communities (ARIC) study, we tested the hypothesis that early age of menarche was associated with higher prevalence of type 2 diabetes at baseline, and higher incidence of diabetes over 9 years of follow-up. We evaluated this association separately in African-American and white women, and whether any associations were independent of measures of adiposity.

\section{Methods}

Participants The ARIC study is a prospective cohort of 15,792 participants $(8,710$ women) aged $45-65$ years at baseline, originally designed to identify risk factors for atherosclerosis and cardiovascular disease. The details of the study cohort, including eligibility criteria, sources and methods of recruitment and follow-up, have been described in detail elsewhere [22]. Briefly, participants were selected from four communities in the USA: Forsyth County, NC $(n=4,035)$; Jackson, MS (black only, $n=3,728)$; northwest suburbs of Minneapolis, MN ( $n=4,009)$; and Washington County, MD $(n=4,020)$. Data for analyses were collected during home interviews and clinic visits at the baseline examination (1987-1989) and three follow-up visits (1990-1992, 1993-1995 and 1996-1998). The overall response rate in the four communities ranged from $80 \%$ to $93 \%$ at the follow-up visits. The mean follow-up time for our analysis was 7.1 years. The study was approved by the institutional review boards at each of the study sites and informed consent was obtained from each participant.

For our study we excluded women with missing age at menarche $(n=49)$, or who reported their age at menarche as $<8$ years $(n=1)$ or $>18$ years $(n=11)$ as we were interested in studying women within the normal range of menarcheal age. Other exclusions included women with race other than white or African-American $(n=25)$, those with missing diabetes status at baseline $(n=87)$, those with diabetes diagnosed before the age of 30 years $(n=30)$ and AfricanAmerican women at the Minneapolis or Washington County sites $(n=16)$ because of small numbers at these two sites. For incident case analyses, we also excluded women with diabetes at baseline $(n=990)$. Our final cohort consisted of 8,491 women ( $n=2,505$ African-American, $n=5,986$ white) for prevalent case analyses and 7,501 women $(n=1,997$ African-American, $n=5,504$ white) for incidence analyses. Follow-up was to the year 1998.

Data collection Age at menarche, defined as age in whole years at the first menstrual period, was self-reported and ascertained during the baseline visit. The validity of recall for age at menarche in middle age is moderate $(r=0.67)$ [23]. In one longitudinal study, approximately $84 \%$ of women, mean age 50 years, were able to recall their age at menarche to within 1 year of the actual date [23, 24].

Diabetes status was assessed at baseline and at each ARIC follow-up clinic visit. We defined type 2 diabetes using blood glucose measurements and self-reported physician diagnoses at each visit. Specifically, diabetes was defined as self-reported history of physician-diagnosed diabetes, fasting glucose $\geq 7.0 \mathrm{mmol} / \mathrm{l}(126 \mathrm{mg} / \mathrm{dl})$, non-fasting glucose $>11.1 \mathrm{mmol} / \mathrm{l}(200 \mathrm{mg} / \mathrm{dl})$ or self-reported use of hypoglycaemic medication in the 2 weeks before the visit for a maximum of 9 years of follow-up.

Prevalent type 2 diabetes was defined as the presence of diabetes at the baseline visit and self-reported age at physician diagnosis 30 years or older. We excluded participants whose self-reported age of diabetes diagnosis was earlier than 30 years because we were concerned that these women may have had type 1 diabetes. For incident type 2 diabetes during 9 years of follow-up, we ascertained the date of onset by imputing the midpoint of the interval between the clinic visit at which diabetes was first discovered and the most recent clinic visit at which diabetes status was ascertained, similar to a previously described method [25]. Fasting glucose was measured by a hexokinase/glucose-6-phosphate dehydrogenase method at the Central Clinical Chemistry Laboratory in Minneapolis, MN, USA, and the Central Lipid Laboratory in Houston, TX, USA.

The covariates of age at baseline, parental history of diabetes, oral contraceptive use, smoking status, level of 
education and weight at age 25 years were all ascertained by self-report via questionnaire at the baseline visit. Birthweight and parental level of education were self-reported at the fourth clinic visit. Weight, height and waist circumference were measured at the clinic visits with participants dressed in scrub suits and shoes removed. Baseline BMI was calculated as baseline weight $(\mathrm{kg}) /$ baseline height ${ }^{2}$ $\left(\mathrm{m}^{2}\right)$. BMI at age 25 years was calculated as self-reported weight at age $25(\mathrm{~kg}) /$ baseline height ${ }^{2}\left(\mathrm{~m}^{2}\right)$.

Statistical analysis We summarised the characteristics of the cohort with unadjusted means and standard deviations for continuous variables and percentages for categorical data. The $p$ value for trend for continuous variables was estimated using the F test type III in linear regression models. Logistic regression was used to evaluate trends in categorical baseline characteristics by age at menarche category. $p$ values were adjusted for baseline age. We used logistic regression to estimate ORs for prevalent diabetes at baseline (diabetes yes/no), and Cox proportional hazards models to estimate the HRs for incident type 2 diabetes by age at menarche. As there was evidence of non-linearity, the $p$ values reported for the relationship between age at menarche and type 2 diabetes were computed with age at menarche represented in the models as a categorical variable $(8-11,12,13,14$ and $15-18$ years, with age 13 years, the median for both race/ethnicity groups, as the reference category). We included an interaction term (race $\times$ menarche age category) to assess interaction by race for early menarche (8-11 vs 13 years) in the models. For Cox models, we tested the proportional hazards assumption by generating a time-dependent predictor and creating interactions between age at menarche and a log function of survival time in the model [26]. All statistical tests were two-sided and significance was set at $\alpha=0.05$.

We ran logistic and Cox proportional hazards models in a series, beginning with a crude model, and then adjusting for age at baseline (years) and ARIC centre in Model 1. For Model 2, we added the potential confounders of parental history of diabetes (yes/no), smoking (current/former/ never), participant education level ( $<$ high school, high school, >high school) and use of oral contraceptives (yes/ no). In our adiposity-adjusted models, we first added earlylife adiposity (BMI at age 25 years) (Model 3), and finally height $(\mathrm{cm})$ and later-life adiposity variables (baseline BMI and waist circumference [cm]) (Model 4).

Because of a large proportion of missing values $(>30 \%)$, we could not include the early-life covariates of birthweight $(\mathrm{g})$ and parental education level (<eighth grade, >eighth grade) in a final model, but rather we added them to Model 4 in sensitivity analyses that included the subset of the cohort that had data available for these variables.

\section{Results}

Study cohort Among the 8,491 women included in our analysis, mean age at menarche was 12.9 years (median 13 years, SD 1.6), and did not differ between white and African-American women (white, median 12.9 years, SD 1.6; African-American, median 12.9 years, SD 1.7; $t$ test $=$ $0.82, p=0.41$ ). Tables 1 and 2 show the characteristics for the cohort by menarche age category. For white women (Table 1), there was a significant trend towards higher percentages of parental history of diabetes and use of hormone replacement therapy, lower participant education level and higher paternal education level with decreasing age at menarche. Younger age at menarche among white women was also associated with younger age at baseline, higher adiposity, higher fasting glucose at baseline and shorter stature. For African-American women (Table 2), younger age at menarche was associated with younger age at baseline, higher adiposity, higher fasting glucose and shorter stature.

Age at menarche and prevalent diabetes After exclusions, there were 990 prevalent type 2 diabetes cases at baseline ( $n=508$ African-American, $n=482$ white). Mean age at diagnosis was 50.6 years, SD 9.3 (African-American, mean 49.8 years, SD 9.0; white, mean 51.3 years, SD 9.0). Among those free of type 2 diabetes at baseline, $16.8 \%$ reported early menarche ( $8-11$ years) vs $22.0 \%$ among those with diabetes at baseline. Before adjustment for covariates, the prevalence of diabetes differed significantly by age at menarche category for white women $\left(\chi^{2}[d f 4, N=5,986]=\right.$ $22.5, p<0.001)$, but not for African-American women $\left(\chi^{2}[d f\right.$ $4, N=2,505]=6.6, p=0.159)$. There was some evidence for interaction between early menarche (8-11 vs 13 years) and race for prevalent diabetes at baseline in the age-adjusted and ARIC centre-adjusted model (Model 1) $(p=0.043)$ and the fully adjusted model (Model 4) $(p=0.093)$. Addition of the interaction term slightly improved the $-2 \log$ likelihood, a measure of model fit, thus supporting our race-stratified analyses.

Table 3 shows the ORs for prevalent diabetes at baseline by menarche age category for white and African-American participants. Adjusting for age and centre, early menarche (8-11 years) was associated with a $72 \%$ increased odds of prevalent diabetes at baseline compared with menarche at 13 years for white participants (OR 1.72, 95\% CI 1.32, 2.25). The results were attenuated after adjustment for lifestyle and adiposity characteristics (Model 4), but remained significant (OR 1.41, 95\% CI 1.05, 1.89). Early menarche was not found to be associated with odds of diabetes for African-American women (Model 1 OR 1.13, 95\% CI 0.84, 1.51; Model 4 OR 0.94, 95\% CI 0.68, 1.30). AfricanAmerican women with menarche at age 14 years (vs 
Table 1 Characteristics of white women in the ARIC study population, by age at menarche category

\begin{tabular}{|c|c|c|c|c|c|c|c|c|c|c|c|}
\hline \multirow[t]{2}{*}{ Age at menarche (years) } & \multicolumn{2}{|c|}{$\begin{array}{l}8-11, n=1,033 \\
(17.2 \%)\end{array}$} & \multicolumn{2}{|c|}{$\begin{array}{l}12, n=1,462 \\
(24.5 \%)\end{array}$} & \multicolumn{2}{|c|}{$\begin{array}{l}13, n=1,25 \\
(28.9 \%)\end{array}$} & \multicolumn{2}{|c|}{$\begin{array}{l}14, n=879 \\
(14.7 \%)\end{array}$} & \multicolumn{2}{|c|}{$\begin{array}{l}15-18, n=887 \\
(14.8 \%)\end{array}$} & \multirow[t]{2}{*}{$p_{\text {trend }}{ }^{\mathrm{a}}$} \\
\hline & $\%$ & Mean (SD) & $\%$ & Mean (SD) & $\%$ & Mean (SD) & $\%$ & Mean (SD) & $\%$ & Mean (SD) & \\
\hline Age (years) & & $53.2(5.5)$ & & $53.9(5.7)$ & & $53.9(5.7)$ & & $54.5(5.7)$ & & $54.7(5.8)$ & $<0.001$ \\
\hline BMI $\left(\mathrm{kg} / \mathrm{m}^{2}\right)$ & & $27.9(6.1)$ & & $26.9(5.4)$ & & $26.4(5.4)$ & & $26.1(5.2)$ & & $25.7(5.0)$ & $<0.001$ \\
\hline Waist circumference $(\mathrm{cm})$ & & $96.0(16.2)$ & & $93.6(15.0)$ & & $92.4(14.5)$ & & $92.1(14.2)$ & & $91.8(14.4)$ & $<0.001$ \\
\hline BMI at age 25 years $\left(\mathrm{kg} / \mathrm{m}^{2}\right)$ & & $22.8(3.8)$ & & $22.2(3.2)$ & & $21.7(3.2)$ & & $21.5(3.0)$ & & $21.2(3.1)$ & $<0.001$ \\
\hline Height (cm) & & $161.0(5.9)$ & & $162.0(5.7)$ & & $162.2(6.0)$ & & $162.1(6.1)$ & & $162.1(6.0)$ & $<0.001$ \\
\hline Glucose (mmol/l) & & $5.9(1.9)$ & & $5.8(1.9)$ & & $5.7(1.7)$ & & $5.6(1.5)$ & & $5.7(1.7)$ & 0.001 \\
\hline $\begin{array}{l}\text { Parental history of } \\
\text { diabetes (yes) }\end{array}$ & 28.4 & & 24.8 & & 23.4 & & 24.0 & & 22.2 & & 0.003 \\
\hline Current smoker (yes) & 29.1 & & 22.7 & & 23.3 & & 24.4 & & 27.3 & & 0.994 \\
\hline $\begin{array}{l}\text { Ever used hormone } \\
\text { replacement therapy }\end{array}$ & 57.4 & & 55.1 & & 54.6 & & 53.9 & & 50.7 & & 0.039 \\
\hline Ever used oral contraceptives & 49.9 & & 46.2 & & 49.5 & & 45.5 & & 39.8 & & 0.085 \\
\hline Education $^{\mathrm{b}}$ & & & & & & & & & & & 0.007 \\
\hline$<$ High school & 18.3 & & 14.5 & & 14.4 & & 16.9 & & 20.6 & & \\
\hline High school & 45.6 & & 43.1 & & 42.4 & & 37.8 & & 32.7 & & \\
\hline$>$ High school & 36.1 & & 42.4 & & 43.1 & & 45.3 & & 46.7 & & \\
\hline $\begin{array}{l}\text { Birthweight }(\mathrm{g}) \\
(n=2,612)\end{array}$ & & $\begin{array}{l}3,139.5 \\
\quad(718.6)\end{array}$ & & $\begin{array}{l}3,207.7 \\
\quad(734.1)\end{array}$ & & $\begin{array}{l}3,159.0 \\
(688.4)\end{array}$ & & $\begin{array}{l}3,178.8 \\
\quad(744.8)\end{array}$ & & $\begin{array}{l}3,097.9 \\
(773.0)\end{array}$ & 0.208 \\
\hline $\begin{array}{l}\text { Mother's education }{ }^{c} \\
(n=4,402)\end{array}$ & & & & & & & & & & & 0.315 \\
\hline$<$ Eighth grade & 38.6 & & 43.4 & & 42.7 & & 43.7 & & 44.0 & & \\
\hline Eighth + grade & 61.5 & & 56.6 & & 57.3 & & 56.3 & & 56.0 & & \\
\hline $\begin{array}{l}\text { Father's education }^{c} \\
\quad(n=4,241)\end{array}$ & & & & & & & & & & & 0.014 \\
\hline$<$ Eighth grade & 43.9 & & 46.1 & & 49.2 & & 50.8 & & 50.9 & & \\
\hline Eighth + grade & 56.1 & & 53.9 & & 50.8 & & 49.2 & & 49.1 & & \\
\hline
\end{tabular}

Linear (continuous variables) or logistic (categorical variables) regression was used to evaluate trends by age at menarche category

${ }^{a}$ All $p$ values are adjusted for age at baseline, except for baseline age

${ }^{\mathrm{b}}$ Average age for high school graduates in the USA is 18 years

${ }^{\mathrm{c}}$ Average age for eighth grade in the USA is 14 years

menarche at 13 years) had $35 \%$ lower odds for diabetes at baseline after adjustment for lifestyle and adiposity-related characteristics (OR $0.65,95 \%$ CI $0.45,0.94)$.

Age at menarche and incident diabetes During an average follow-up time of 7.1 years, we identified 755 incident cases of type 2 diabetes ( $n=322$ African-American, $n=433$ white). Mean age at diagnosis for incident diabetes was 56.8 years, SD 8.0 (African-American, 56.3 years, SD 5.9; white, 57.2 years, SD 9.1). There was no evidence of interaction between early age at menarche $(8-11$ vs 13 years) and race in the incidence models (Model 1: interaction, $p=0.527$, Model 4: interaction, $p=0.554$ ). However, we ran race-stratified models because our a priori research question was to assess race-specific associations. Among white women, early age at menarche (8-11 years) was associated with a $43 \%$ increased risk of diabetes during follow-up (HR 1.43, 95\% CI 1.08, 1.89) compared with menarche at age 13 years after adjusting for age and ARIC centre (Model 1) (Table 4). The statistically significant association remained after adjustment for family history of diabetes, smoking status, oral contraceptive use and level of education (Model 2). The association was no longer significant after adjusting for early-life and laterlife adiposity measures as the CIs crossed unity (Models 3 and 4). Of note is that there was a suggestion of a U-shaped relationship between age at menarche and incident diabetes. The HR for diabetes among those with late age at menarche (15-18 years) vs menarche at 13 years was similar to the HR for early menarche after adjustment for 
Table 2 Characteristics of African-American women in the ARIC study population, by age at menarche category

\begin{tabular}{|c|c|c|c|c|c|c|c|c|c|c|c|}
\hline \multirow[t]{2}{*}{ Age at menarche (years) } & \multicolumn{2}{|c|}{$\begin{array}{l}8-11, n=444 \\
(17.6 \%)\end{array}$} & \multicolumn{2}{|c|}{$\begin{array}{l}12, n=591 \\
(23.6 \%)\end{array}$} & \multicolumn{2}{|c|}{$\begin{array}{l}13, n=671 \\
(26.8 \%)\end{array}$} & \multicolumn{2}{|c|}{$\begin{array}{l}14, n=367 \\
(14.7 \%)\end{array}$} & \multicolumn{2}{|c|}{$\begin{array}{l}15-18, n=432 \\
(17.3 \%)\end{array}$} & \multirow[t]{2}{*}{$p_{\text {trend }}{ }^{\mathrm{a}}$} \\
\hline & $\%$ & Mean (SD) & $\%$ & Mean (SD) & $\%$ & Mean (SD) & $\%$ & Mean (SD) & $\%$ & Mean (SD) & \\
\hline Age (years) & & $52.9(5.5)$ & & $53.4(5.7)$ & & $53.4(5.7)$ & & $54.0(5.9)$ & & $53.7(5.9)$ & $<0.001$ \\
\hline BMI $\left(\mathrm{kg} / \mathrm{m}^{2}\right)$ & & $31.6(6.1)$ & & $30.7(6.6)$ & & $30.7(6.6)$ & & $30.4(6.3)$ & & $30.0(6.3)$ & 0.004 \\
\hline Waist circumference $(\mathrm{cm})$ & & $102.6(15.6)$ & & $99.9(16.5)$ & & $99.9(16.5)$ & & $100.1(16.5)$ & & $99.1(16.6)$ & 0.007 \\
\hline BMI at age $25\left(\mathrm{~kg} / \mathrm{m}^{2}\right)$ & & $23.6(5.3)$ & & $22.4(4.0)$ & & $22.4(4.0)$ & & $21.8(3.3)$ & & $21.7(4.4)$ & $<0.001$ \\
\hline Height $(\mathrm{cm})$ & & $162.5(6.2)$ & & $162.7(6.2)$ & & $163.4(6.4)$ & & $163.3(6.3)$ & & $164.0(6.0)$ & 0.001 \\
\hline Glucose $(\mathrm{mmol} / \mathrm{l})$ & & $7.0(3.8)$ & & $6.6(3.3)$ & & $6.6(3.3)$ & & $6.3(2.7)$ & & $6.7(3.5)$ & 0.012 \\
\hline $\begin{array}{l}\text { Parental history of } \\
\text { diabetes (yes) }\end{array}$ & 28.9 & & 33.2 & & 26.7 & & 31.1 & & 27.1 & & 0.574 \\
\hline Current smoker (yes) & 26.1 & & 25.9 & & 24.4 & & 20.2 & & 26.0 & & 0.449 \\
\hline $\begin{array}{l}\text { Ever used hormone } \\
\text { replacement therapy }\end{array}$ & 39.8 & & 39.3 & & 40.7 & & 42.3 & & 34.0 & & 0.348 \\
\hline Ever used oral contraceptives & 42.1 & & 43.0 & & 40.4 & & 37.3 & & 37.5 & & 0.472 \\
\hline Education $^{\mathrm{b}}$ & & & & & & & & & & & 0.642 \\
\hline$<$ High school & 42.1 & & 39.7 & & 37.3 & & 43.4 & & 40.1 & & \\
\hline High school & 35.5 & & 35.9 & & 40.8 & & 33.6 & & 37.0 & & \\
\hline$>$ High school & 22.4 & & 24.4 & & 21.9 & & 23.0 & & 22.9 & & \\
\hline $\begin{array}{l}\text { Birthweight }(\mathrm{g}) \\
(n=535)\end{array}$ & & $\begin{array}{l}3,125.3 \\
(888.4)\end{array}$ & & $\begin{array}{l}3,198.2 \\
\quad(698.8)\end{array}$ & & $\begin{array}{l}3,226.3 \\
(696.6)\end{array}$ & & $\begin{array}{l}3,206.8 \\
(932.9)\end{array}$ & & $\begin{array}{l}3,212.6 \\
\quad(901.7)\end{array}$ & 0.936 \\
\hline \multicolumn{12}{|l|}{ Mother's education ${ }^{\mathrm{c}}$} \\
\hline$(n=1,249)$ & & & & & & & & & & & 0.463 \\
\hline$<$ eighth grade & 59.8 & & 58.3 & & 64.5 & & 61.7 & & 55.6 & & \\
\hline Eighth + grade & 40.2 & & 41.8 & & 35.6 & & 38.3 & & 44.4 & & \\
\hline \multicolumn{12}{|l|}{ Father's education ${ }^{\mathrm{c}}$} \\
\hline$(n=358)$ & & & & & & & & & & & 0.395 \\
\hline$<$ Eighth grade & 66.5 & & 67.6 & & 69.9 & & 65.6 & & 64.3 & & \\
\hline Eighth + grade & 33.5 & & 32.4 & & 30.1 & & 34.4 & & 35.7 & & \\
\hline
\end{tabular}

Linear (continuous variables) or logistic (categorical variables) regression was used to evaluate trends by age at menarche category

${ }^{a}$ All $p$ values are adjusted for age at baseline, except for baseline age

${ }^{\mathrm{b}}$ Average age for high school graduates in the USA is 18 years

${ }^{\mathrm{c}}$ Average age for eighth grade in the USA is 14 years

adiposity, although not statistically significant (HR 1.29, 95\% CI $0.95,1.73$ ).

We did not find an association between age at menarche and incident diabetes among African-American women (Model 1 HR 1.20, 95\% CI 0.87, 1.67; Model 4 HR 1.11, $95 \%$ CI $0.80,1.56)$. Nonetheless, the direction of the association was similar to the results in white women.

Sensitivity analysis In sensitivity analyses for both prevalent and incident cases, we found that the addition of birthweight, mother's education and father's education to Model 4, for the subset of the cohort that had this information available, did not substantially change the associations in either the white women or the AfricanAmerican women.

\section{Discussion}

In the ARIC cohort, early age at menarche was associated with increased risk for type 2 diabetes among white women. The association was stronger for prevalent diabetes at baseline than for incident diabetes during 9 years of follow-up. Associations were attenuated after adjustment for adiposity. We did not find an association among African-American women.

Past studies have been inconsistent regarding the association of age at menarche and diabetes [1-4, 27, 28]. Those studies that reported an association tended to be large and included both premenopausal and postmenopausal women [1-4], while those that did not find an association included only postmenopausal women and might have been underpowered [27, 28]. In all studies, 
Table 3 OR of prevalent diabetes at baseline according to age at menarche category, ARIC study, 1987-1989

\begin{tabular}{|c|c|c|c|c|c|c|c|c|c|}
\hline \multirow[t]{2}{*}{ Age at menarche (years) } & \multicolumn{2}{|l|}{$8-11$} & \multicolumn{2}{|l|}{12} & \multirow{2}{*}{$\frac{13}{\text { Reference }}$} & \multicolumn{2}{|l|}{14} & \multicolumn{2}{|c|}{$15-18$} \\
\hline & OR & $95 \% \mathrm{CI}$ & OR & $95 \% \mathrm{CI}$ & & OR & $95 \% \mathrm{CI}$ & OR & $95 \% \mathrm{CI}$ \\
\hline \multicolumn{10}{|l|}{ All women } \\
\hline$n$ & & 1,477 & & 2,053 & 2,396 & & 1,246 & & 1,319 \\
\hline No. of cases & & 218 & & 231 & 268 & & 117 & & 156 \\
\hline Model 1 & 1.42 & $1.17,1.73$ & 1.01 & $0.84,1.23$ & 1.00 & 0.77 & $0.61,0.98$ & 0.95 & $0.77,1.18$ \\
\hline Model 2 & 1.37 & $1.12,1.68$ & 0.96 & $0.79,1.17$ & 1.00 & 0.71 & $0.56,0.90$ & 0.92 & $0.74,1.14$ \\
\hline Model 3 & 1.23 & $1.00,1.52$ & 0.93 & $0.76,1.14$ & 1.00 & 0.74 & $0.58,0.95$ & 0.97 & $0.78,1.23$ \\
\hline Model 4 & 1.20 & $0.97,1.48$ & 0.92 & $0.75,1.13$ & 1.00 & 0.73 & $0.57,0.94$ & 0.97 & $0.77,1.23$ \\
\hline \multicolumn{10}{|l|}{ White women } \\
\hline$n$ & & 1,033 & & 1,462 & 1,725 & & 879 & & 887 \\
\hline No. of cases & & 117 & & 124 & 124 & & 54 & & 63 \\
\hline Model 1 & 1.72 & $1.32,2.25$ & 1.18 & $0.91,1.54$ & 1.00 & 0.81 & $0.58,1.13$ & & $0.66,1.24$ \\
\hline Model 2 & 1.69 & $1.29,2.23$ & 1.17 & $0.90,1.52$ & 1.00 & 0.77 & $0.55,1.09$ & 0.87 & $0.63,1.20$ \\
\hline Model 3 & 1.51 & $1.14,2.00$ & 1.11 & $0.85,1.46$ & 1.00 & 0.80 & $0.57,1.13$ & 0.93 & $0.67,1.30$ \\
\hline Model 4 & 1.41 & $1.05,1.89$ & 1.12 & $0.85,1.48$ & 1.00 & 0.81 & $0.57,1.15$ & 0.93 & $0.67,1.31$ \\
\hline \multicolumn{10}{|l|}{ African-American women } \\
\hline$n$ & & 444 & & 591 & 671 & & 367 & & 432 \\
\hline No. of cases & & 101 & & 107 & 144 & & 63 & & 93 \\
\hline Model 1 & 1.13 & $0.84,1.51$ & 0.85 & $0.64,1.13$ & 1.00 & 0.73 & $0.52,1.02$ & 0.98 & $0.73,1.32$ \\
\hline Model 2 & 1.06 & $0.79,1.44$ & 0.76 & $0.57,1.01$ & 1.00 & 0.64 & $0.45,0.90$ & 0.94 & $0.69,1.28$ \\
\hline Model 3 & 0.95 & $0.70,1.31$ & 0.74 & $0.55,1.00$ & 1.00 & 0.67 & $0.47,0.96$ & 0.99 & $0.71,1.36$ \\
\hline Model 4 & 0.94 & $0.68,1.30$ & 0.72 & $0.53,0.99$ & 1.00 & 0.65 & $0.45,0.94$ & 0.97 & $0.70,1.36$ \\
\hline
\end{tabular}

Model 1: adjusted for age at baseline visit, race (for models that include all women) and centre

Model 2: adjusted for variables in Model 1 plus family history of diabetes, smoking status (current, former, never), use of oral contraceptives and participant education level ( $<$ high school, high school, $>$ high school)

Model 3: adjusted for variables in Model 2 plus BMI at age 25 years

Model 4: adjusted for variables in Model 3 plus baseline BMI, baseline height and baseline waist circumference

associations were attenuated by adiposity to varying degrees. It is possible that the association itself varies over the course of a lifetime, and the role of obesity may depend on the definitions of diabetes and adiposity used by researchers.

Our finding of an association among white women is in agreement with four recent studies [1-4]. For example, women in the US Nurses' Health Study (NHS) I with menarche age 11 years or younger (vs age 13 years) had a $34 \%$ increased risk for diagnosed type 2 diabetes (HR 1.34, 95\% CI 1.26, 1.42) [1]. After adjustment for BMI and other social and lifestyle factors, the risk was $18 \%$ higher (HR 1.18, 95\% CI 1.10, 1.27). In the NHS II, which followed a younger cohort of women, those with menarche at or before 11 years (vs age 13 years) had a $98 \%$ increased risk for type 2 diabetes (HR 1.98, 95\% CI 1.79, 2.20 ) before adjustment for adult BMI, and $40 \%$ (HR 1.40, $95 \%$ CI 1.24, 1.57) increased risk after adjustment for adiposity and other potential confounders [1]. In the EPIC (European Prospective Investigation of Cancer)-Norfolk study, the odds for diagnosed diabetes at baseline or during 8 years of follow- up were reduced by $10 \%$ (OR $0.90,95 \%$ CI $0.95,0.86$ ) for each 1-year delay in menarche [2]. Similar to our findings for incident diabetes during follow-up, the association was completely attenuated and no longer significant after adjustment for adult BMI ( $2 \%$ reduced odds for each year delay in menarche [OR 0.98, 95\% CI 0.97, 1.03]). The German Cooperative Health Research in the Region of Augsburg (KORA) study found that the RR for diabetes per 1 year delay in menarche was nearly the same before and after adjustment for BMI and other confounders (unadjusted RR 0.83, 95\% CI 0.73, 0.95; BMI-adjusted RR 0.84, 95\% CI 0.73, 0.98) [3]. Finally, a study that followed women from birth to age 53 years found that age at menarche was associated with diabetes before, but not after, adjustment for BMI (unadjusted RR per year delay in menarche 0.73, 95\% CI 0.56, 0.96; adjusted for BMI RR $0.85,95 \%$ CI $0.65,1.10)$.

Previous studies on the relationship of age at menarche and diabetes have included primarily white populations. Thus, little is known about the relationship in non-white populations. In our analyses of the biethnic ARIC 
Table 4 HR of incident diabetes according to age at menarche category, ARIC study, 1987-1998

\begin{tabular}{|c|c|c|c|c|c|c|c|c|c|}
\hline \multirow[t]{2}{*}{ Age at menarche (years) } & \multicolumn{2}{|l|}{$8-11$} & \multicolumn{2}{|l|}{12} & \multirow{2}{*}{$\frac{13}{\text { Reference }}$} & \multicolumn{2}{|l|}{14} & \multicolumn{2}{|c|}{$15-18$} \\
\hline & HR & $95 \% \mathrm{CI}$ & HR & $95 \% \mathrm{CI}$ & & HR & $95 \% \mathrm{CI}$ & HR & $95 \% \mathrm{CI}$ \\
\hline \multicolumn{10}{|l|}{ All women } \\
\hline$n$ & & 1,259 & & 1,822 & 2,128 & & 1,129 & & 1,163 \\
\hline No. of cases & & 151 & & 178 & 192 & & 108 & & 126 \\
\hline Model 1 & 1.32 & $1.06,1.63$ & 1.06 & $0.87,1.30$ & 1.00 & 1.04 & $0.82,1.32$ & 1.15 & $0.92,1.44$ \\
\hline Model 2 & 1.27 & $1.02,1.58$ & 1.03 & $0.83,1.26$ & 1.00 & 0.97 & $0.76,1.23$ & 1.15 & $0.92,1.44$ \\
\hline Model 3 & 1.20 & $0.97,1.50$ & 1.00 & $0.81,1.23$ & 1.00 & 0.98 & $0.77,1.24$ & 1.20 & $0.96,1.51$ \\
\hline Model 4 & 1.18 & $0.95,1.47$ & 1.01 & $0.82,1.24$ & 1.00 & 1.00 & $0.78,1.27$ & 1.24 & $0.98,1.55$ \\
\hline \multicolumn{10}{|l|}{ White women } \\
\hline$n$ & & 916 & & 1,338 & 1,601 & & 825 & & 824 \\
\hline No. of cases & & 88 & & 99 & 110 & & 62 & & 74 \\
\hline Model 1 & 1.43 & $1.08,1.89$ & 1.06 & $0.81,1.39$ & 1.00 & 1.08 & $0.78,1.47$ & 1.28 & $0.95,1.71$ \\
\hline Model 2 & 1.38 & $1.04,1.83$ & 1.05 & $0.80,1.38$ & 1.00 & 1.04 & $0.76,1.41$ & 1.23 & $0.91,1.65$ \\
\hline Model 3 & 1.30 & $0.98,1.73$ & 1.03 & $0.78,1.35$ & 1.00 & 1.06 & $0.77,1.44$ & 1.26 & $0.94,1.70$ \\
\hline Model 4 & 1.22 & $0.92,1.63$ & 1.03 & $0.78,1.35$ & 1.00 & 1.08 & $0.79,1.48$ & 1.29 & $0.95,1.73$ \\
\hline \multicolumn{10}{|l|}{ African-American women } \\
\hline$n$ & & 343 & & 484 & 527 & & 304 & & 339 \\
\hline No. of cases & & 63 & & 79 & 82 & & 46 & & 52 \\
\hline Model 1 & 1.20 & $0.87,1.67$ & 1.05 & $0.77,1.44$ & 1.00 & 0.98 & $0.68,1.40$ & 0.99 & $0.70,1.40$ \\
\hline Model 2 & 1.14 & $0.82,1.60$ & 0.99 & $0.73,1.36$ & 1.00 & 0.89 & $0.62,1.28$ & 0.99 & $0.70,1.40$ \\
\hline Model 3 & 1.11 & $0.79,1.55$ & 0.98 & $0.72,1.35$ & 1.00 & 0.88 & $0.61,1.28$ & 1.05 & $0.74,1.49$ \\
\hline Model 4 & 1.11 & $0.80,1.56$ & 0.99 & $0.72,1.36$ & 1.00 & 0.88 & $0.61,1.29$ & 1.06 & $0.74,1.51$ \\
\hline
\end{tabular}

Model 1: adjusted for age at baseline visit, race (for models that include all women) and centre

Model 2: adjusted for variables in Model 1 plus family history of diabetes, smoking status (current, former, never), use of oral contraceptives and participant education level (<high school, high school, $>$ high school)

Model 3: adjusted for variables in Model 2 plus BMI at age 25 years

Model 4: adjusted for variables in Model 3 plus baseline BMI, baseline height and baseline waist circumference

population, we found no association between age at menarche and diabetes among African-American women. Biases or low statistical power might account for the lack of association, as the results were consistent with a slightly higher risk among African-American women with early menarche. However, because African-American women had a higher prevalence of diabetes, the power was greater for the prevalent analyses, and only slightly lower for the incident analyses, compared with white women (see electronic supplementary material [ESM] Figs. 1, 2). Alternatively, early menarche may not be a risk factor for type 2 diabetes for African-American women. We found a significant interaction between early age at menarche and race for prevalent diabetes at baseline, indicating possible race/ethnic differences in the association during early adulthood. As discussed previously, timing of menarche is likely a marker for early childhood exposures that, in general, influence type 2 diabetes risk. In an analysis of the association between leg length (also a marker for early-life environment) and risk for incident diabetes in the ARIC cohort, shorter leg length was associated with increased diabetes risk for white, but not African-American, participants [25]. Most African-Americans were enrolled at a single site, and it is possible that African-American women in our cohort experienced more homogenous early-life exposures to factors that influence diabetes risk, such as nutrition levels and socioeconomic status (SES) [25], making it difficult to detect differences in the association between the various menarcheal age categories and diabetes. In addition, BMI and waist circumference at baseline and weight gain since age 25 years were much higher for African-American women than for white women. In our cohort, waist circumference and BMI were all stronger risk factors for diabetes than age at menarche (data not shown), and so it is possible that the high prevalence of obesity in the African-American women in our study masked the more subtle influence of early menarche on diabetes risk.

We noted a few differences between our results for prevalent diabetes at baseline and incident diabetes during follow-up. First, although both analyses showed an association among white women before adjustment for adiposity, 
after adjustment the association with incident diabetes was no longer statistically significant. One reason for this might be low power of detecting the relatively small effect sizes in the incidence analysis after adjustment for adiposity. The second difference was that the ORs for prevalent diabetes were stronger than the HRs for incident diabetes. ORs tend to be more extreme than measures of risk when an outcome is relatively common $(>10 \%)$ [29]; the prevalence of diabetes was approximately $12 \%$ at baseline. Also, the prevalence analysis included younger women compared with the incidence analysis, and data from the NHS I and II support a stronger association in younger compared with older women [1]. Furthermore, the prevalence analysis was subject to a number biases, including survival bias, to which the incidence analysis was less susceptible. The third main difference is that we found an interaction between race and age at menarche for prevalent diabetes at baseline, but not incident diabetes during follow-up. It may be that there are true race/ ethnic differences in the association at younger vs older ages, or the interaction might reflect differences in survival between African-American and white women.

The possible biological pathways for an association of early menarche and type 2 diabetes fit well into a 'developed country' model, in which the parents' generation has relatively higher SES compared with earlier generations, leading to more rapid childhood growth and adiposity in offspring, which in turn leads to earlier menarche and even greater adiposity, and then insulin resistance and eventually type 2 diabetes. As discussed in earlier studies [1, 2], an independent association between early menarche and diabetes is biologically plausible by means of hormone exposure and/or changes in glucose metabolism that track into adulthood and have been shown to be a factor in type 2 diabetes pathogenesis [1, 30-34].

Although not hypothesised in advance, the risk for incident diabetes among the late menarche group (15-18 years) was similar to that among the early menarche group, but the associations were not statistically significant. This finding may merit investigation in future studies. The later-maturing women in our study had lower adiposity at age 25 years and at baseline compared with the reference group (menarche= 13 years), and one would expect lower diabetes risk in unadjusted models if obesity is the main pathway through which earlier age at menarche increases diabetes risk. Weight gain from age 25 years to baseline was slightly greater for women with menarche at $15-18$ vs 13 years (15.1 vs $14.7 \mathrm{~kg}$, respectively), and the effects of weight gain on diabetes risk may differ by menarche category. Late menarche has been associated with lower SES and larger family size in other studies $[35,36]$. Poor nutrition and/or a more stressful childhood environment may suppress the hypothalamic-pituitary-gonadal axis and trigger later age at menarche [17]. Notably, the women taking part in the
ARIC study were born between 1924 and 1944. Many lived through the US Great Depression and as a consequence may have been exposed to poor nutrition during their early life. The 'thrifty phenotype hypothesis' [17] states that poor nutritional environment during fetal and infant development leads to programming for later chronic disease, such as type 2 diabetes, through changes in the glucose-insulin metabolism. Additionally, both low SES and under-nutrition during fetal development, possibly mediated by rapid infant weight gain [37], and later exposure to more plentiful diets place individuals at higher risk of diabetes in adulthood [38, 39].

We acknowledge that there are weaknesses in our analysis. Bias is possible if error in the recall of menarche age was differential according to diabetes status. We have no reason to believe that women with diabetes recalled age at menarche any differently to those without diabetes and such bias would be unlikely in our analysis of incident type 2 diabetes. Nondifferential misclassification of menarche age and covariates might have biased associations, likely toward the null, possibly explaining lack of association among AfricanAmerican women. Counter to findings from more recent studies, average age at menarche for African-American and white women in the ARIC study did not differ. This raises concern about potential selection bias among AfricanAmerican women if those with younger age at menarche were less likely to enrol in ARIC compared with their latermaturing counterparts. However, many of the women in the ARIC study were born during the 1930s and studies reporting earlier average age at menarche for African-American women included more recent cohorts. It is unknown when race/ethnic differences in average menarche age began to appear. BMI at age 25 years was similar for white and African-American women in the ARIC study. Given that age at menarche and BMI are strongly associated, this suggests that there might not have been race/ethnic differences in average menarcheal age earlier in the 20th century. Furthermore, general health status, hospitalisation, and prevalence of myocardial infarction, stroke or diabetes did not differ among African-American responders and non-responders, supporting minimal influence of selection bias on our result [40]. Another weakness was inadequate information on those childhood risk factors that may be associated with both the timing of menarche and chronic disease risk. In addition, despite our large sample size, for some stratified analyses, there were few events and CIs were wide, making it difficult to detect an effect for modest associations. Finally, misclassification of cases was possible because our definition for diabetes was less stringent than that used in clinical practice, which requires confirmation of elevated glucose or presence of symptoms [41, 42]. Such misclassification was likely non-differential and unlikely to have an impact on OR or HR estimates.

The strengths of our analysis were the inclusion of AfricanAmerican women, large sample size and both cross-sectional 
and prospective analyses. Another strength was that we were able to include both diagnosed and undiagnosed type 2 diabetes cases at the time of each visit because type 2 diabetes status was ascertained by blood glucose measurements as well as by self-reported physician diagnosis. We adjusted for a wide range of confounding factors, including BMI in early and late adulthood, and waist circumference at baseline.

In conclusion, our results support recent studies that show early age at menarche is associated with an increased risk for type 2 diabetes among white women and that adiposity attenuates the association. We did not find a similar association among African-American women, although possible biases might have accounted for the lack of association. Nonetheless, our findings suggest that there may be race/ethnic differences in the influence of developmental factors in the aetiology of type 2 diabetes, which merit further investigation. Studies in younger women, ideally starting before menarche and even prenatally, are needed to clarify the role of childhood adiposity and other early-life exposures on menarche age and subsequent type 2 diabetes risk.

Acknowledgements The ARIC study is a collaborative study supported by the National Heart, Lung, and Blood Institute contracts (HHSN268201100005C, HHSN268201100006C, HHSN268201100007C, HHSN268201100008C, HHSN268201100009C, HHSN268201100010C, HHSN268201100011C and HHSN268201100012C). The authors thank the staff and participants of the ARIC study for their important contributions.

Research reported in this publication was supported by the National Heart, Lung, and Blood Institute of the National Institutes of Health under Award Number T32HL007779 (JGD). The content is solely the responsibility of the authors and does not necessarily represent the official views of the National Institutes of Health.

Parts of this study were presented in abstract form at the American Heart Association, Cardiovascular Disease Epidemiology and Prevention 2011 Scientific Sessions, Atlanta, GA, Mar 22-25, 2011.

Duality of interest The authors declare that there is no duality of interest associated with this manuscript.

Contribution statement JGD designed the analytic strategy, undertook analyses, interpreted results, and wrote, reviewed and edited the manuscript. PLL, JSP, NF, RH, LFR, ES and EWD contributed to the study design, results interpretation and manuscript revision. All authors provided final approval of the manuscript.

\section{References}

1. He C, Zhang C, Hunter DJ et al (2010) Age at menarche and risk of type 2 diabetes: results from 2 large prospective cohort studies. Am J Epidemiol 171:334-344

2. Lakshman R, Forouhi N, Luben R et al (2008) Association between age at menarche and risk of diabetes in adults: results from the EPIC-Norfolk cohort study. Diabetologia 51:781-786

3. Stockl D, Doring A, Peters A et al (2012) Age at menarche is associated with prediabetes and diabetes in women (aged 32-81 years) from the general population: the KORA F4 Study. Diabetologia 55:681-688
4. Pierce MB, Kuh D, Hardy R (2011) The role of BMI across the life course in the relationship between age at menarche and diabetes, in a British Birth Cohort. Diabet Med 29:600-603

5. Euling SY, Herman-Giddens ME, Lee PA et al (2008) Examination of US puberty-timing data from 1940 to 1994 for secular trends: panel findings. Pediatrics 121(Suppl 3):S172-S191

6. Freedman DM, Tarone RE, Doody MM et al (2002) Trends in reproductive, smoking, and other chronic disease risk factors by birth cohort and race in a large occupational study population. Ann Epidemiol 12:363-369

7. Centers for Disease Control and Prevention. (2010) Long-term Trends in diabetes. National Diabetes Surveillance System, CDC's Division of Diabetes Translation. Available from www.cdc.gov/ diabetes/statistics, accessed 22 February 2012

8. Ahmed ML, Ong KK, Dunger DB (2009) Childhood obesity and the timing of puberty. Trends Endocrinol Metabol 20:237-242

9. Himes JH (2006) Examining the evidence for recent secular changes in the timing of puberty in US children in light of increases in the prevalence of obesity. Mol Cell Endocrinol 254-255:13-21

10. Himes JH, Obarzanek E, Baranowski T, Wilson DM, Rochon J, McClanahan BS (2004) Early sexual maturation, body composition, and obesity in African-American girls. Obes Res 12 (Suppl):64S-72S

11. Demerath EW, Li J, Sun SS et al (2004) Fifty-year trends in serial body mass index during adolescence in girls: the Fels Longitudinal Study. Am J Clin Nutr 80:441-446

12. Garn SM, Haskell JA (1960) Fat thickness and developmental status in childhood and adolescence. AMA J Dis Child 99:746-751

13. Adair LS, Gordon-Larsen P (2001) Maturational timing and overweight prevalence in US adolescent girls. Am J Public Health 91:642-644

14. Kivimaki M, Lawlor DA, Smith GD et al (2008) Association of age at menarche with cardiovascular risk factors, vascular structure, and function in adulthood: the Cardiovascular Risk in Young Finns study. Am J Clin Nutr 87:1876-1882

15. Demerath EW, Towne B, Chumlea WC et al (2004) Recent decline in age at menarche: the Fels Longitudinal Study. Am J Hum Biol $16: 453-457$

16. de Ridder CM, Thijssen JH, Bruning PF, van den Brande JL, Zonderland ML, Erich WB (1992) Body fat mass, body fat distribution, and pubertal development: a longitudinal study of physical and hormonal sexual maturation of girls. J Clin Endocrinol Metab $75: 442-446$

17. Karapanou O, Papadimitriou A (2010) Determinants of menarche. Reprod Biol Endocrinol 8:115

18. Freedman DS, Khan LK, Serdula MK, Dietz WH, Srinivasan SR, Berenson GS (2002) Relation of age at menarche to race, time period, and anthropometric dimensions: the Bogalusa Heart Study. Pediatrics 110:e43

19. Brancati FL, Kao WH, Folsom AR, Watson RL, Szklo M (2000) Incident type 2 diabetes mellitus in African American and white adults: the Atherosclerosis Risk in Communities Study. JAMA 283:2253-2259

20. Shai I, Jiang R, Manson JE et al (2006) Ethnicity, obesity, and risk of type 2 diabetes in women: a 20 -year follow-up study. Diabetes Care 29:1585-1590

21. Katzmarzyk PT, Bray GA, Greenway FL et al (2010) Racial differences in abdominal depot-specific adiposity in white and African American adults. Am J Clin Nutr 91:7-15

22. The Atherosclerosis Risk in Communities (ARIC) (1989) Study: design and objectives. The ARIC investigators. Am J Epidemiol 129:687-702

23. Casey VA, Dwyer JT, Coleman KA, Krall EA, Gardner J, Valadian I (1991) Accuracy of recall by middle-aged participants in a longitudinal study of their body size and indices of maturation earlier in life. Ann Hum Biol 18:155-166 
24. Coleman L, Coleman J (2002) The measurement of puberty: a review. J Adolesc 25:535-550

25. Weitzman S, Wang CH, Pankow JS, Schmidt MI, Brancati FL (2010) Are measures of height and leg length related to incident diabetes mellitus? The ARIC (Atherosclerosis Risk in Communities) study. Acta Diabetol 47:237-242

26. UCLA: Academic Technology Services SCG (2011) Introduction to SAS. Available from www.ats.ucla.edu/stat/sas/notes, accessed 20 April 2011

27. Saquib N, Kritz-Silverstein D, Barrett-Connor E (2005) Age at menarche, abnormal glucose tolerance and type 2 diabetes mellitus: the Rancho Bernardo Study. Climacteric 8:76-82

28. Cooper GS, Ephross SA, Sandler DP (2000) Menstrual patterns and risk of adult-onset diabetes mellitus. J Clin Epidemiol 53:1170-1173

29. Vittinghoff EGD, Shiboski SC, McCulloch CE (2004) Regression methods in biostatitistics: linear, logistic, survival, and repeated measures models. Springer, New York

30. Apter D, Reinila M, Vihko R (1989) Some endocrine characteristics of early menarche, a risk factor for breast cancer, are preserved into adulthood. Int J Cancer 44:783-787

31. Ibanez L, Potau N, Chacon P, Pascual C, Carrascosa A (1998) Hyperinsulinaemia, dyslipaemia and cardiovascular risk in girls with a history of premature pubarche. Diabetologia 41:10571063

32. Apter D, Bolton NJ, Hammond GL, Vihko R (1984) Serum sex hormone-binding globulin during puberty in girls and in different types of adolescent menstrual cycles. Acta Endocrinol (Copenh) 107:413-419

33. Ding EL, Song Y, Malik VS, Liu S (2006) Sex differences of endogenous sex hormones and risk of type 2 diabetes: a systematic review and meta-analysis. JAMA 295:1288-1299
34. Remsberg KE, Demerath EW, Schubert CM, Chumlea WC, Sun SS, Siervogel RM (2005) Early menarche and the development of cardiovascular disease risk factors in adolescent girls: the Fels Longitudinal Study. J Clin Endocrinol Metab 90:2718-2724

35. Wronka I, Pawlinska-Chmara R (2005) Menarcheal age and socioeconomic factors in Poland. Ann Hum Biol 32:630-638

36. Morris DH, Jones ME, Schoemaker MJ, Ashworth A, Swerdlow AJ (2010) Determinants of age at menarche in the UK: analyses from the Breakthrough Generations study. Br J Cancer 103:1760-1764

37. dos Santos SI, de Stavola BL, Mann V, Kuh D, Hardy R, Wadsworth ME (2002) Prenatal factors, childhood growth trajectories and age at menarche. Int J Epidemiol 31:405-412

38. Li Y, He Y, Qi L et al (2010) Exposure to the Chinese famine in early life and the risk of hyperglycemia and type 2 diabetes in adulthood. Diabetes 59:2400-2406

39. de Lauzon-Guillain B, Balkau B, Charles MA, Romieu I, BoutronRuault MC, Clavel-Chapelon F (2010) Birth weight, body silhouette over the life course, and incident diabetes in 91,453 middleaged women from the French Etude Epidemiologique de Femmes de la Mutuelle Generale de l'Education Nationale (E3N) Cohort. Diabetes Care 33:298-303

40. Jackson R, Chambless LE, Yang K et al (1996) Differences between respondents and nonrespondents in a multicenter community-based study vary by gender ethnicity. The Atherosclerosis Risk in Communities (ARIC) Study Investigators. J Clin Epidemiol 49:1441-1446

41. American Diabetes Association (2012) Diagnosis and classification of diabetes mellitus. Diabetes Care 35:S65-S71

42. World Health Organization (1999) Definition, diagnosis, and classification of diabetes mellitus and its complications. World Health Organization, Department of Noncommunicable Disease Surveillance, Geneva 